\title{
Three ancient problems solved by using the game theory logic based on the Shapley value
}

\author{
Silviu Guiasu
}

Received: 30 March 2010 / Accepted: 13 September 2010 / Published online: 25 September 2010

(C) Springer Science+Business Media B.V. 2010

\begin{abstract}
The ancient problems of bankruptcy, contested garment, and rights arbitration have generated many studies, debates, and controversy. The objective of this paper is to show that the Shapley value from game theory, measuring the power of each player in a game, may be consistently applied for getting the general one-step solution of all these three problems viewed as $n$-person games. The decision making is based on the same tool, namely the game theory logic based on the use of the Shapley value, but the specific games involved are slightly different in each problem. The kind of claims of the players, the relationship between the given claims and the given resources available, and the particular way of calculating the generalized characteristic function of the game determine the specific type of game which has to be solved in each of the three ancient problems mentioned. The iterative use of the Shapley value may also justify the well-known Aumann-Maschler step-by-step procedure for solving the bankruptcy problem.
\end{abstract}

Keywords Bankruptcy problem · Contested garment problem · Rights arbitration problem - Shapley value - Cumulative and maximal generalized characteristic functions

\section{Introduction}

Three ancient problems, whose intriguing solutions were given without any justification back then, generated many studies with very different interpretations, debates, and controversy. Heuristic procedures and new concepts have been introduced, just to

\section{S. Guiasu ( $\varangle)$}

York University, Toronto, ON, Cananda

e-mail: guiasus@pascal.math.yorku.ca 
justify the numerical solutions given very long ago, but a uniform method for solving these three ancient problems was still missing.

\subsection{The bankruptcy problem}

As mentioned by Gura (2009), in the ancient bankruptcy problem, known also as the Talmudic problem of three wives, a man married three women and promised them in their marriage contract the sum of $d_{1}=100, d_{2}=200$, and $d_{3}=300$ units of money to be given to them upon his death. The man died but his estate $E$ amounted to less then 600 units. The Mishna, attributed to Rabbi Nathan, treats the cases in which the estate $E$ was worth 100,200 , and 300 units of money. The division of the estate among the three wives, corresponding to these three cases, is shown in Table 1 .

In the modern law, a division of the estate proportionally with the corresponding debts is the common practice. Table 1 shows both a uniform distribution of the estate (for $E=100$ ), and a proportional distribution (for $E=300$ ), whereas the distribution corresponding to $E=200$ has raised question marks and objections about how it was obtained and how it could be rationalized and justified. As noted by Aumann and Maschler (1985): "The figures for $E=200$ look mysterious; but whatever they may mean, they do not fit any obvious extension of either equal or proportional division. A common rationale for all three cases is not apparent. Over two millennia, [the data set from Table 1] has spawned a large literature. Many authorities disagree with it outright. Others attribute the figures to special circumstances not made explicit [in the Talmud]. A few have attempted direct rationalization of the figures as such, mostly with little success. One modern scholar, exasperated by his inability to make sense of the text, suggested errors in transcription. In brief, the passage [i.e. Table 1] is notoriously difficult." In the end, Aumann and Maschler (1985) proposed the following heuristic procedure for justifying the solutions for the bankruptcy problem from Table 1: Assuming that there are $n$ creditors and that the estate satisfies the inequality $E \leq\left(d_{1}+\cdots+d_{n}\right) / 2$, for instance, "when [the estate] $E$ is small, all $n$ claimants divide it equally. This continues until 1 has received $d_{1} / 2$; for the time being [claimant 1] stops receiving payments, and each additional dollar is divided equally between the remaining $n-1$ claimants. This, in turn, continues until claimant 2 has received $d_{2} / 2$, at which point [claimant 2] stops receiving payments for the time being, and each additional dollar is divided equally between the remaining $n-2$ claimants. The process continues until each claimant has received half his claim. This happens when $E=\left(d_{1}+\cdots+d_{n}\right) / 2$." This procedure

Table 1 Bankruptcy problem in the Babylonian Talmud

\begin{tabular}{llll}
\hline Estate & Debt & & \\
\cline { 2 - 4 } & 100 & 200 & 300 \\
\hline 100 & $33 \frac{1}{3}$ & $33 \frac{1}{3}$ & $33 \frac{1}{3}$ \\
200 & 50 & 75 & 75 \\
300 & 50 & 100 & 150 \\
\hline
\end{tabular}


Table 2 Contested garment problem in the Babylonian Talmud

Table 3 Rights arbitration problem

\begin{tabular}{lll}
\hline Estate & Claim & \\
\cline { 2 - 3 } & $1 / 2$ & 1 \\
\hline 1 & $1 / 4$ & $3 / 4$ \\
\hline
\end{tabular}

\begin{tabular}{|c|c|c|c|c|}
\hline \multirow[t]{2}{*}{ Estate } & \multicolumn{4}{|c|}{ Claim } \\
\hline & 30 & 40 & 60 & 120 \\
\hline 120 & $7 \frac{1}{2}$ & $10 \frac{5}{6}$ & $20 \frac{5}{6}$ & $80 \frac{5}{6}$ \\
\hline
\end{tabular}

works for explaining heuristically how the solutions from Table 1 (for $E=200$ and $E=300$ ) and Table 2 may be obtained. The entire procedure seems to favour the creditors with smaller claims. But no justification was given why a small estate $E=100$ has to be divided equally among claimants, and why the claimants whose claims are smaller than the available resources are successively awarded half of their respective claims. Also, if this procedure is applied to the rights arbitration problem, the results are completely different from those given in Table 3.

\subsection{The contested garment problem}

This is another ancient problem from the Babylonian Talmud which, according to O'Neill (1982), was formulated in a very simple way: "Two hold a garment. If one of them says, 'It is all mine', and the other says, 'Half of it is mine', then the former receives three quarters and the latter receives one quarter." How can such a solution, shown in Table 2, be justified?

\subsection{The rights arbitration problem}

According to O’Neill (1982), around 1140 A.D., Abraham Ibn Ezra formulated the following inheritance problem: "Jacob died and his son Reuben produced a deed duly witnessed that Jacob willed to him the entire estate on his death, son Simeon also produced a deed that his father willed to him half of the estate, Levi produced a deed giving him one third and Judah brought forth a deed giving one quarter. All of them bear the same date." The wills seem equally valid but are mutually inconsistent because they give away more than the total estate. Assuming that the estate is $E=120$, in whatever units, Ibn Ezra mentioned that one solution would be to divide the estate in proportion to each son's claim but rejected such a proportional solution in favour of another one, according to which the four sons Judah, Levi, Simeon, and Reuben have to get: $7 \frac{1}{2}, 10 \frac{5}{6}, 20 \frac{5}{6}$, and $80 \frac{5}{6}$, respectively, as shown in Table 3.

The general decision process of arbitration is very complex and the decision maker, called here the judge, can be very subjective in his final ruling. The many ways of 
solving the bankruptcy and rights arbitration problems are amply presented in the extensive survey paper Thomson (2003). Here, on the other hand, we focus only on the three ancient problems just mentioned and the objective is to show that the Shapley value (Shapley 1953) from the $n$-person game theory, measuring the power of the different players involved in a game, can be used for justifying, in a uniform and unambiguous way, the ancient solutions given to all these three problems. In the next section, the Shapley value method is used for getting the general solution, in one step, of these three ancient problems viewed as $n$-person games.

It is not without interest to note that the concept of Shapley value was mentioned both in the context of the bankruptcy problem in Aumann and Maschler (1985), Moulin (1992), Moulin (2003), Thomson (2003), Alcade et al. (2005), De Mesnard (2008), and in the analysis of the rights arbitration problem (O'Neill 1982). In particular, (Moulin 2003 , p. 57) pointed clearly that the Shapley value applied to a two-claimant game gives the same solution as that of the contested garment problem. On the other hand, Aumann and Maschler (1985) showed that the games corresponding to the bankruptcy problem for $E=200$ and $E=300$ may be solved by applying the solution of the contested garment problem to each pair of contestants. However, the game corresponding to the bankruptcy problem for the case $E=100$ and the game corresponding to the rights arbitration problem cannot be solved by using the solution of the contested garment problem. O'Neill (1982) also discussed several decision methods and proposed the method of recursive completion which is in fact the Shapley value of the cooperative game corresponding to the bankruptcy problem. Recently, De Mesnard (2008) showed that the Shapley value method applied to the bankruptcy problem, viewed as a regular three-person game, gives a solution for $E=200$ different from the Talmudic solution, implying the conclusion that the use of the Shapley value cannot fully justify all the ancient solutions shown in Tables $1-3$. The present paper arrives to a different conclusion.

The objective of this paper is to show that the Shapley value from game theory, measuring the power of each player in a game, may be consistently applied for getting the general one-step solution of all these three problems, namely the bankruptcy problem, the contested garment problem, and the rights arbitration problem, viewed as $n$-person games. The decision making is based on the same tool, namely the game theory logic based on the iterative use of the Shapley value, but the specific games involved are slightly different in each problem. The kind of claims of the players, the relationship between the given claims and the given resources available, and the particular way of calculating the generalized characteristic function of the game determine the specific type of game which has to be solved in each of the three ancient problems mentioned. Ignoring the specific kind of game induced by the relationship between the values of the claims and the value of the estate involved in each problem could give misleading results like De Mesnard (2008) counterexample which seemed to compromise the possibility of solving the bankruptcy problem as a three-person game by using the Shapley value in the case $E=200$.

The Shapley value may be calculated only if we know the generalized characteristic function of the respective game. The delicate point is that the given claims and the estate do not determine completely the values of the generalized characteristic function for the proper possible coalitions of the game. This opens the door to different 
ways for filling this gap and, therefore, to different possible solutions of the game. This paper shows that the division of the estate from Tables 1-3 may be obtained by using only the Shapley value, as a mathematical tool, if the bankruptcy problem is viewed as being a cumulative game and the rights arbitration problem is viewed as being a maximal game. A cumulative game is the classic case of an $n$-person game in which the values of the characteristic function for the proper coalitions are calculated additively, reflecting the fact that the members are not willing to reach a compromise and share their claims. A maximal game is the classic case of an $n$-person game in which the values of the characteristic function for the proper coalitions are calculated by taking the maximum claim into account, reflecting the fact that the members are willing to reach a compromise and share their claims.

Section 2 contains the general one-step solutions of the three ancient problems viewed as $n$-person games, using the Shapley value method. Section 3 shows that the well-known Aumann and Maschler (1985) solutions for the bankruptcy and contested garment problems may be obtained and justified by an iterative application of the Shapley value method when, at each step, the creditor with the lowest claim competes against all the other remaining creditors.

\section{General, one-Step solutions of the ancient problems viewed as $\boldsymbol{n}$-person games}

\subsection{Shapley value}

Let $N=\{1, \ldots, n\}$ be the set of players. Every subset $S \subseteq N$ is a possible coalition. There are $2^{n}$ possible subsets $S$ of $N$, including the empty set $\emptyset$. To give an $n$-person game means to give the set $N$ of players and, for each subset $S \subseteq N$, a real number $v(S)$ representing the value of the coalition $S$, such that:

$$
v(\emptyset)=0, \quad v(S) \geq 0, \quad v\left(S_{1}\right) \leq v\left(S_{2}\right) \quad \text { if } \quad S_{1} \subset S_{2} .
$$

In an $n$-person game with the generalized characteristic function $v$, the amount $v(S \cup\{i\})-v(S)$ measures the contribution of player $\{i\}$ to the increase in the value of coalition $S$ if this player joins in. The Shapley value is the average of players' marginal contributions over all permutations. Thus, the Shapley value of player $i$ is:

$$
\omega_{i}=\sum_{S \subset N, i \notin S}[v(S \cup\{i\})-v(S)] p_{n}(S),
$$

where:

$$
p_{n}(S)=\frac{|S| !(n-|S|-1) !}{n !}
$$

and $|S|$ is the number of players from the coalition $S \subseteq N$. The Shapley value shows the power of the respective player (Shapley 1953; Winston 1994). 
The nonnegative monotonic set function $v$ is called the generalized characteristic function of the game. The monotonicity property simply states that if a coalition $S_{2}$ contains more players than coalition $S_{1}$, then $S_{2}$ cannot have a smaller value than $S_{1}$. The set function $v$, satisfying (1), is called the generalized characteristic function because, normally, it is required to be a supperadditive set function, which means that the value of a union of disjoint coalitions, $v\left(S_{1} \cup S_{2}\right)$ is supposed to be larger than or equal to $v\left(S_{1}\right)+v\left(S_{2}\right)$. If a nonnegative set function is superadditive then, obviously, it is monotonic. The converse is not necessarily true. One of the qualities of the Shapley value over other solutions of an $n$-person game is that it uses only the monotonicity of $v$ and not superadditivity.

\subsection{The games induced by the claims of the players and the estate}

Let us assume that we have a set of players, $N=\{1, \ldots, n\}$. Their claims are $d_{1}<$ $\cdots<d_{n}$, and the total resource available is the estate $E<\left(d_{1}+\cdots+d_{n}\right) / 2$. The assumption that the claims are different, as it is the case in the three ancient problems, is not essential but simplifies the analysis.

If the values $v(\{1\}), \ldots, v(\{n\})$ of the generalized characteristic function $v$ assigned to each player are given, then the corresponding $n$-person game is cumulative if the value of the generalized characteristic function $v$ for each subset of players $\left\{i_{1}, \ldots, i_{k}\right\}$ is calculated according to:

$$
v\left(\left\{i_{1}, \ldots, i_{k}\right\}\right)=\min \left\{v\left(\left\{i_{1}\right\}\right)+\cdots+v\left(\left\{i_{k}\right\}\right), E\right\},
$$

for all $k=2, \ldots, n$. In such a game the claim of each coalition is the sum of the claims of its members if it does not exceed the estate $E$. It corresponds to the case when the players stick to their own claims and are not willing to compromise and share the claims.

If the values $v(\{1\}), \ldots, v(\{n\})$ of the generalized characteristic function $v$ assigned to each player are given, then the corresponding $n$-person game is maximal if the value of the generalized characteristic function $v$ for each subset of players $\left\{i_{1}, \ldots, i_{k}\right\}$ is calculated according to:

$$
v\left(\left\{i_{1}, \ldots, i_{k}\right\}\right)=\min \left\{\max \left\{v\left(\left\{i_{1}\right\}\right), \ldots, v\left(\left\{i_{k}\right\}\right)\right\}, E\right\},
$$

for all $k=2, \ldots, n$. In such a game the claim of each coalition is the maximum of the claims of its members if it does not exceed the estate $E$. It corresponds to the case when the players are willing to compromise and share the maximum claim.

In our context, in a cumulative game the claim of a coalition is the sum of the claims of its members or the entire estate if this sum exceeds the estate. In such a game, the members of a coalition make no compromise; each sticks to his own claim and is not willing to share parts of it with other members of the respective coalition. The members of a coalition expect the judge to give them their respective claim. In a maximal game, the claim of a coalition is the maximum claim of its members and they are supposed to share this maximum among them. In such a game, the judge expects 
the members of a coalition to cooperate and reach a compromise, sharing the amount awarded to the respective coalition.

The division of the estate in the three wives problem, as given in Table 1, may be justified if the judge looks at the corresponding game as being a cumulative game with the wives unwilling to compromise and share parts of their claims.

The division of the estate in the rights arbitration problem, as given in Table 3, may be justified if the judge looks at the corresponding game as being a maximal game, assuming that the four brothers are willing to reach a compromise and share parts of their claims when they form a coalition.

On the other hand, the contested garment problem is very simple. The two claims $d_{1}, d_{2}$ and the estate $E$ completely determine the corresponding game because there are no proper coalitions in the total set $N=\{1,2\}$.

Remark Let us take an arbitrary cumulative or maximal game in which the generalized characteristic function $v$ assigns to each player his claim, if it does not exceed the estate, namely $v(\{i\})=\min \left\{d_{i}, E\right\},(i=1, \ldots, n)$. If $E \leq d_{1}<\cdots<d_{n}$, which means that no claim is smaller than the estate, then, according to their Shapley values, the players get the same reward $\omega_{i}=E / n,(i=1, \ldots, n)$, sharing the estate $E$.

This result, which is true both for cumulative and maximal games, is often accepted as being obvious; nevertheless, it may be justified using the Shapley value and plays an important role in what follows. Indeed, if the game is cumulative,

$$
\begin{gathered}
v(\{i\})=\min \left\{d_{i}, E\right\}=E, \quad(i=1, \ldots, n) \\
v\left(\left\{i_{1}, \ldots, i_{k}\right\}\right)=\min \left\{v\left(\left\{i_{1}\right\}\right)+\cdots+v\left(\left\{i_{k}\right\}\right), E\right\}=E, \quad(k=2, \ldots, n) .
\end{gathered}
$$

If the game is maximal,

$$
\begin{gathered}
v(\{i\})=\min \left\{d_{i}, E\right\}=E, \quad(i=1, \ldots, n) \\
v\left(\left\{i_{1}, \ldots, i_{k}\right\}\right)=\min \left\{\max \left\{v\left(\left\{i_{1}\right\}\right), \ldots, v\left(\left\{i_{k}\right\}\right)\right\}, E\right\}=E, \quad(k=2, \ldots, n) .
\end{gathered}
$$

The Shapley value of player $i$ is:

$$
\omega_{i}=(E-0) p_{n}(\emptyset)+(E-E) \sum_{S \subset N, i \notin S, S \neq \emptyset} p_{n}(S)=E 0 !(n-0-1) ! / n !=E / n
$$

The result just discussed shows that if a player wants to get the entire estate when his claim exceeds the estate, cannot do this because if no claim is smaller than the current estate available, it has to be shared equally among the players. Based on this essential fact, we define the generalized characteristic function of the game such that the value which is assigned to a player reflects the maximum claim he can ask for, subject to the restrictions induced by the relationship between all the claims and the portion of the estate available. Taking into account the result just proved, when we define the generalized characteristic function of the game, we have to make a distinction between the players whose claims are smaller than the estate and those whose claims are not smaller than the estate. 
Based on this essential remark, we set the following axiom: In the general case, if $d_{1}<\cdots<d_{m-1}<E \leq d_{m}<\cdots<d_{n}$, we define:

$$
\begin{gathered}
v(\emptyset)=0, \quad v(\{i\})=d_{i}, \quad(i=1, \ldots, m-1), \\
v(\{i\})=d_{m-1}+\left(E-d_{m-1}\right) /(n-m+1), \quad(i=m, \ldots, n) .
\end{gathered}
$$

According to (4), the generalized characteristic function $v$ assigns the value $d_{i}$ to the player $i$, if his claim is smaller than the estate, whereas, according to (5), the other $n-m+1$ players whose claims exceed the estate $E$, cannot claim more than $d_{m-1}$ plus the excess of the resource $E-d_{m-1}$ equally divided among them, as shown at the beginning of this Subsection.

\subsection{Solution of the bankruptcy problem}

There are three players, $N=\{1,2,3\}$, and we have three possible cases which generalize the Talmudic variants of the bankruptcy problem.

Case 1 If $E \leq d_{1}<d_{2}<d_{3}$, we have $n=3$ and $m=1$. According to the remark discussed at the beginning of Sect. 2.2, the Shapley value implies that the solution is:

$$
\omega_{1}=E / 3, \quad \omega_{2}=E / 3, \quad \omega_{3}=E / 3 .
$$

In the Talmudic problem, $E=100, d_{1}=100, d_{2}=200, d_{3}=300$ and the solution (6) becomes: $\omega_{1}=\omega_{2}=\omega_{3}=100 / 3=33(1 / 3)$.

Case 2 If $d_{1}<E \leq d_{2}<d_{3}$, we have $n=3$ and $m=2$. According to (4) and (5), the generalized characteristic function of the corresponding three-person cumulative game has the values:

$$
\begin{gathered}
v(\emptyset)=0, \quad v(\{1\})=d_{1}, \quad v(\{2\})=d_{1}+\left(E-d_{1}\right) / 2, \quad v(\{3\})=d_{1}+\left(E-d_{1}\right) / 2, \\
v(\{1,2\})=\min \left\{d_{1}+d_{2}, E\right\}=E, \quad v(\{1,3\})=\min \left\{d_{1}+d_{3}, E\right\}=E, \\
v(\{2,3\})=\min \left\{d_{2}+d_{3}, E\right\}=E, \quad v(\{1,2,3\})=\min \left\{d_{1}+d_{2}+d_{3}, E\right\}=E .
\end{gathered}
$$

For this three-person game, the Shapley values of the players are:

$$
\begin{aligned}
\omega_{1}= & \left(d_{1}-0\right)(2 / 6)+\left[\left(E-d_{1}\right) / 2\right](1 / 6)+\left[\left(E-d_{1}\right) / 2\right](1 / 6)+(E-E)(2 / 6) \\
= & \left(E+d_{1}\right) / 6 . \\
\omega_{2}= & \omega_{3}=\left\{\left[d_{1}+\left(E-d_{1}\right) / 2\right]-0\right\}(2 / 6)+\left(E-d_{1}\right)(1 / 6) \\
& +\left[E-d_{1}-\left(E-d_{1}\right) / 2\right](1 / 6)+(E-E)(2 / 6)=\left(5 E-d_{1}\right) / 12,
\end{aligned}
$$

Obviously, $\omega_{1}+\omega_{2}+\omega_{3}=E$.

Therefore, the solution is:

$$
\omega_{1}=\left(E+d_{1}\right) / 6, \quad \omega_{2}=\left(5 E-d_{1}\right) / 12, \quad \omega_{3}=\left(5 E-d_{1}\right) / 12
$$


In the Talmudic problem, $E=200, d_{1}=100, d_{2}=200, d_{3}=300$ and the solution (7) becomes: $\omega_{1}=50, \omega_{2}=75, \omega_{3}=75$.

Case 3 If $d_{1}<d_{2}<E \leq d_{3}<$, we have $n=3$ and $m=3$. According to (4) and (5), the generalized characteristic function of the corresponding three-person cumulative game has the values:

$$
\begin{aligned}
& v(\emptyset)=0, \quad v(\{1\})=d_{1}, \quad v(\{2\})=d_{2}, \quad v(\{3\})=d_{2}+\left(E-d_{2}\right) / 1=E, \\
& v(\{1,2\})=\min \left\{d_{1}+d_{2}, E\right\}=\left\{\begin{array}{lll}
d_{1}+d_{2}, & \text { if } & d_{1}+d_{2}<E, \\
E, & \text { if } & d_{1}+d_{2} \geq E,
\end{array}\right. \\
& v(\{1,3\})=\min \left\{d_{1}+d_{3}, E\right\}=E, \\
& v(\{2,3\})=\min \left\{d_{2}+d_{3}, E\right\}=E, \quad v(\{1,2,3\})=\min \left\{d_{1}+d_{2}+d_{3}, E\right\}=E .
\end{aligned}
$$

(a) Let us assume that $d_{1}+d_{2}<E$. Then, in this three-person game, the Shapley values of the players are:

$$
\begin{aligned}
\omega_{1}= & \left(d_{1}-0\right)(2 / 6)+\left[\left(d_{1}+d_{2}\right)-d_{2}\right](1 / 6)+(E-E)(1 / 6)+(E-E)(2 / 6)=d_{1} / 2, \\
\omega_{2}= & \left(d_{2}-0\right)(2 / 6)+\left[\left(d_{1}+d_{2}\right)-d_{1}\right](1 / 6)+(E-E)(1 / 6)+(E-E)(2 / 6)=d_{2} / 2, \\
\omega_{3}= & (E-0)(2 / 6)+\left(E-d_{1}\right)(1 / 6)+\left(E-d_{2}\right)(1 / 6) \\
& +\left[E-\left(d_{1}+d_{2}\right)\right](2 / 6)=E-\left[\left(d_{1} / 2\right)+\left(d_{2} / 2\right)\right] .
\end{aligned}
$$

Therefore, if $d_{1}+d_{2}<E$, the solution is:

$$
\omega_{1}=d_{1} / 2, \quad \omega_{2}=d_{2} / 2, \quad \omega_{3}=E-\left[\left(d_{1} / 2\right)+\left(d_{2} / 2\right)\right] .
$$

(b) Let us assume that $d_{1}+d_{2} \geq E$. Then, in this three-person game, the Shapley values of the players are:

$$
\begin{aligned}
\omega_{1} & =\left(d_{1}-0\right)(2 / 6)+\left(E-d_{2}\right)(1 / 6)+(E-E)(1 / 6)+(E-E)(2 / 6) \\
& =\left(E+2 d_{1}-d_{2}\right) / 6, \\
\omega_{2} & =\left(d_{2}-0\right)(2 / 6)+\left(E-d_{1}\right)(1 / 6)+(E-E)(1 / 6)+(E-E)(2 / 6) \\
& =\left(E-2 d_{2}-d_{1}\right) / 6, \\
\omega_{3} & =(E-0)(2 / 6)+\left(E-d_{1}\right)(1 / 6)+\left(E-d_{2}\right)(1 / 6)+(E-E)(2 / 6) \\
& =\left(4 E-d_{1}-d_{2}\right) / 6 .
\end{aligned}
$$

Therefore, if $d_{1}+d_{2} \geq E$, the solution is:

$\omega_{1}=\left(E+2 d_{1}-d_{2}\right) / 6, \omega_{2}=\left(E-2 d_{2}-d_{1}\right) / 6, \omega_{3}=\left(4 E-d_{1}-d_{2}\right) / 6$.

In the Talmudic problem, $E=300, d_{1}=100, d_{2}=200, d_{3}=300$ and the solution (9) becomes: $\omega_{1}=50, \omega_{2}=100, \omega_{3}=150$. 
Remarks 1. Each of the solutions (6)-(9) is monotonic because, in each of them, the Shapley values $\omega_{1}, \omega_{2}$, and $\omega_{3}$, when they depend on the estate $E$, are linear functions of $E$, with the coefficients of E positive. Therefore, no player (claimant) loses from an increase in the estate $E$.

2. Each of the solutions (6)-(9) is order-preserving because, in each of them, the Shapley values $\omega_{1}, \omega_{2}$, and $\omega_{3}$, satisfy:

$$
\omega_{1} \leq \omega_{2} \leq \omega_{3}, \quad 0 \leq d_{1}-\omega_{1} \leq d_{2}-\omega_{2} \leq d_{3}-\omega_{3},
$$

showing that a player with a higher claim than another gets an award that is no smaller and suffers a loss that is no smaller; both the awards and the loose preserve the order of the claims.

3. De Mesnard (2008) used the Shapley value for solving the bankruptcy problem as a three-person game assuming, as an axiom, that the values of the generalized characteristic function $v$ are $v(\{i\})=\min \left\{d_{i}, E\right\}$, for all players, making no difference between the players whose claims are smaller than the estate and those whose claims are not smaller than the estate. For the case $E=200$, the solution obtained by $\operatorname{him}\left(\omega_{1}=33 \frac{1}{3}, \omega_{2}=83 \frac{1}{3}, \omega_{3}=83 \frac{1}{3}\right)$ is not the Talmudic solution $\left(\omega_{1}=50, \omega_{2}=75, \omega_{3}=75\right)$, casting a serious doubt over any attempt of getting all the Talmudic solutions using the Shapley value of the corresponding three-person game. Nothing is wrong with De Mesnard's computations and he correctly looked at the corresponding three-person game as being a cumulative game, without explicitly stating this, but he followed Aumann and Maschler (1985) and assumed that the 'truncation' (Moulin 2003, pp. 37-38, 262) is done before sharing, which means that he used a different axiom than that used in this paper.

4. Let us note that $\left(E+d_{1}\right) / 6=d_{1} / 2$ if and only if $E+d_{1}=3 d_{1}$ or $E=2 d_{1}$. This happens in the Talmudic case when $E=200$ and $d_{1}=100$.

\subsection{Solution of the contested garment problem}

There are two players $N=\{1,2\}$ and $d_{1}<E=d_{2}$, which means that $n=2$ and $m=2$. According to (4) and (5), the values of the generalized characteristic function assigned to the two players are:

$$
v(\emptyset)=0, \quad v(\{1\})=d_{1}, \quad v(\{2\})=d_{1}+\left(E-d_{1}\right) / 1=E,
$$

and, if the two-person game is viewed as being either cumulative or maximal, we have:

$$
v(\{1,2\})=\min \left\{d_{1}+E, E\right\}=\min \left\{\max \left\{d_{1}, E\right\}, E\right\}=E .
$$

The Shapley values of the two players are:

$$
\begin{aligned}
& \omega_{1}=\left(d_{1}-0\right)(1 / 2)+(E-E)(1 / 2)=d_{1} / 2, \\
& \omega_{2}=(E-0)(1 / 2)+\left(E-d_{1}\right)(1 / 2)=E-\left(d_{1} / 2\right) .
\end{aligned}
$$


Therefore, the solution of the general contested garment problem is:

$$
\omega_{1}=d_{1} / 2, \quad \omega_{2}=E-\left(d_{1} / 2\right) .
$$

In the Talmudic version, $E=1, d_{1}=1 / 2$, and $d_{2}=E=1$. Introducing these values in (10), we get: $\omega_{1}=1 / 4$ and $\omega_{2}=1-(1 / 4)=3 / 4$.

Remark The solution (10) is obviously monotonic and order-preserving.

\subsection{Solution of the rights arbitration problem}

Let us assume that we have a maximal four-player game where the players are $N=$ $\{1,2,3,4\}$, and the claims are such that $d_{1}<d_{2}<d_{3}<E=d_{4}$. Therefore, $n=4$ and $m=4$. According to (4) and (5), the values of the generalized characteristic function of the game assigned to the players are:

$$
\begin{gathered}
v(\{\emptyset\})=0, \quad v(\{1\})=d_{1}, \quad v(\{2\})=d_{2}, \quad v(\{3\})=d_{3}, \\
v(\{4\})=d_{3}+\left(E-d_{3}\right) / 1=E=d_{4}
\end{gathered}
$$

The game being maximal, the values of the generalized characteristic function for the proper subsets of players are:

$$
\begin{aligned}
& v(\{1,2\})=\min \left\{\max \left\{d_{1}, d_{2}\right\}, E\right\}=d_{2}, \\
& v(\{1,3\})=\min \left\{\max \left\{d_{1}, d_{3}\right\}, E\right\}=d_{3}, \\
& v(\{1,4\})=\min \left\{\max \left\{d_{1}, d_{4}\right\}, E\right\}=d_{4}=E, \\
& v(\{2,3\})=\min \left\{\max \left\{d_{2}, d_{3}\right\}, E\right\}=d_{3}, \\
& v(\{2,4\})=\min \left\{\max \left\{d_{2}, d_{4}\right\}, E\right\}=d_{4}=E, \\
& v(\{3,4\})=\min \left\{\max \left\{d_{3}, d_{4}\right\}, E\right\}=d_{4}=E, \\
& v(\{1,2,3\})=\min \left\{\max \left\{d_{1}, d_{2}, d_{3}\right\}, E\right\}=d_{3}, \\
& v(\{1,2,4\})=\min \left\{\max \left\{d_{1}, d_{2}, d_{4}\right\}, E\right\}=d_{4}=E, \\
& v(\{1,3,4\})=\min \left\{\max \left\{d_{1}, d_{3}, d_{4}\right\}, E\right\}=d_{4}=E, \\
& v(\{2,3,4\})=\min \left\{\max \left\{d_{2}, d_{3}, d_{4}\right\}, E\right\}=d_{4}=E,
\end{aligned}
$$

Taking into account that:

$$
\begin{gathered}
p_{4}(\emptyset)=0 !(4-0-1) ! / 4 !=1 / 4, \\
p_{4}(\{i\})=p_{4}(\{i, j\})=(1 !)(2 !) / 4 !=1 / 12 \quad \text { if } \quad i=j, \\
p_{4}(\{i, j, k\})=3 !(4-3-1) ! / 4 !=1 / 4 \quad \text { if } \quad i \neq j \neq k,
\end{gathered}
$$

the Shapley values of the players are:

$$
\begin{gathered}
\omega_{1}=d_{1} / 4, \quad \omega_{2}=\left(4 d_{2}-d_{1}\right) / 12, \quad \omega_{3}=\left(6 d_{3}-d_{1}-2 d_{2}\right) / 12, \\
\omega_{4}=\left(12 d_{4}-d_{1}-2 d_{2}-6 d_{3}\right) / 12
\end{gathered}
$$


Going back to the ancient rights arbitration problem from Table 3, the respective claims are: $d_{1}=30, d_{2}=40, d_{3}=60, d_{4}=120$, corresponding to a quarter, a third, a half, and all of the estate $E=120$, respectively. Introducing these values in the general solution (11), (12), we get:

$$
\omega_{1}=7 \frac{1}{2}, \quad \omega_{2}=10 \frac{5}{6}, \quad \omega_{3}=20 \frac{5}{6}, \quad \omega_{4}=80 \frac{5}{6},
$$

which is the Talmudic solution.

\section{Iterative, step-by-step solutions of the ancient problems}

In Sect. 2, the three ancient problems have been solved in one step, viewed as threeperson, two-person, and four-person games, respectively. There is an alternative way of solving them, in an iterative way, following a step-by-step approach.

\subsection{The iterative solution of the bankruptcy problem}

Aumann and Maschler (1985) proposed an iterative, step-by-step solution of the bankruptcy problem, as explained in Sect.1. It is based on two assumptions, taken as postulates:

Assumption 1 If no claim is smaller than the current available estate $\tilde{E}$, each creditor gets $\tilde{E} / n$.

Assumption 2 If $i$ is the first creditor whose claim $d_{i}$ is smaller than the remaining estate available $E_{i-1}$, where $E_{0}$ is the initial estate $E$, he is awarded $d_{i} / 2$; the remaining creditors get $E_{i}=E_{i-1}-d_{i} / 2$.

Except these explicit assumptions, the Aumann-Maschler solution implicitly assumes that the judge gives the awards sequentially, starting with the creditor which has the lowest claim. We notice also that Assumption 2 mentioned above is equivalent to the application of the contested garment solution at each step, as long as there is a claim smaller than the current remaining estate.

The Shapley value method implies the Aumann-Maschler solution. Indeed, as proved in Sect. 2.4, the Shapley value gives the contested garment solution. This fact has been also noticed by (Moulin 2003, p. 57). Therefore, at each step, the creditor with the smallest claim smaller than the current remaining estate available, competes against all the other creditors, taken together, in a two-person game. The Shapley value assigned to him is indeed half of his claim. On the other hand, as shown in the remark from the beginning of Sect. 2.2, the Shapley value justifies Assumption 1, postulated by Aumann-Maschler, showing that if there is no claim smaller than the current available estate, the remaining creditors have to share it equally. 
3.2 The iterative solution of the rights arbitration problem

Let us assume that we have a four-player game where the players are $N=\{1,2,3,4\}$, and the claims are such that $d_{1}<d_{2}<d_{3}<E=d_{4}$. The entire estate may be split in four disjoint parts: $(0, E]=\left(0, d_{1}\right] \cup\left(d_{1}, d_{2}\right] \cup\left(d_{2}, d_{3}\right] \cup\left(d_{3}, d_{4}\right]$.

Assuming that all players are willing to share the portion of the estate to which they are entitled, we have four steps:

Step 1 For the first portion, $E_{1}=d_{1}-0=d_{1}$ and $E_{1}=d_{1}<d_{2}<d_{3}<d_{4}$. According to the remark proved in the beginning in Sect. 2.2, the Shapley value implies the following solution of the corresponding subgame: $\omega_{1,1}=\omega_{2,1}=\omega_{3,1}=\omega_{4,1}=d_{1} / 4$.

Step 2 For the second portion, we have $E_{2}=d_{2}-d_{1}$ and $E_{2}=d_{2}<d_{3}<d_{4}$. As shown in the remark from the beginning of Sect. 2.2, the Shapley value implies the following solution of the corresponding subgame: $\omega_{2,2}=\omega_{3,2}=\omega_{4,2}=\left(d_{2}-d_{1}\right) / 3$.

Step 3 For the third portion, $E_{3}=d_{3}-d_{2}$ and $E_{3}=d_{3}<d_{4}$. As shown in the remark from the beginning of Sect. 2.2, the Shapley value implies the following solution of the corresponding subgame: $\omega_{3,3}=\omega_{4,3}=\left(d_{3}-d_{2}\right) / 2$.

Step 4 For the fourth portion, $E_{4}=d_{4}-d_{3}$ and $E_{4}=d_{4}$. As shown in the remark from the beginning of Sect. 2.2, the Shapley value implies the following solution of the corresponding subgame: $\omega_{4,4}=\left(d_{4}-d_{3}\right) / 1$.

Taking all four steps into account, the iterative solution of the rights arbitration problem is:

$$
\begin{gathered}
\omega_{1}=\omega_{1,1}=d_{1} / 4, \quad \omega_{2}=\omega_{2,1}+\omega_{2,2}=d_{1} / 4+\left(d_{2}-d_{1}\right) / 3 \\
\omega_{3}=\omega_{3,1}+\omega_{3,2}+\omega_{3,3}=d_{1} / 4+\left(d_{2}-d_{1}\right) / 3+\left(d_{3}-d_{2}\right) / 2, \\
\omega_{4}=\omega_{4,1}+\omega_{4,2}+\omega_{4,3}+\omega_{4,4} \\
=d_{1} / 4+\left(d_{2}-d_{1}\right) / 3+\left(d_{3}-d_{2}\right) / 2+\left(d_{4}-d_{3}\right) / 1
\end{gathered}
$$

which is just the solution (11), (12), given in Sect. 2.

Going back to the ancient rights arbitration problem from Table 3, the respective claims are: $d_{1}=30, d_{2}=40, d_{3}=60, d_{4}=120$, corresponding to a quarter, a third, a half, and all of the estate $E=120$, respectively. Introducing these values in the general solution (13)-(15), we get:

$$
\omega_{1}=7 \frac{1}{2}, \quad \omega_{2}=10 \frac{5}{6}, \quad \omega_{3}=20 \frac{5}{6}, \quad \omega_{4}=80 \frac{5}{6},
$$

which is the Talmudic solution.

Remark For the rights arbitration problem with $d_{1}=30, d_{2}=40, d_{3}=60, d_{4}=120$, $E=120$, the Aumann-Maschler solution $\left(\omega_{1}=15, \omega_{2}=20, \omega_{3}=30, \omega_{4}=55\right)$ is different from the Talmudic solution. 


\section{Conclusion}

Today, all the cases mentioned in the three ancient problems would be probably solved using the division of the estate proportionally with the respective claims and perhaps very few people would object to such a solution. But the main purpose of this paper, and of so many other papers about this topic, is to find a rationale for the ancient solutions. The objective of this paper is to show that the game theory logic based on the Shapley value may be consistently applied for solving, in a uniform way, all three ancient problems, namely the three wives problem, the contested garment problem, and the rights arbitration problem. The main conclusion is that using the concept of Shapley value from the $n$-person game theory, all the numerical solutions to these ancient problems, as mentioned in Tables 1-3, may be obtained in a straightforward way. Before using this tool, however, it is essential to identify what kind of games are involved in each of these problems. The decision making is based on the same tool, namely the game theory logic based on the Shapley value, but the specific games involved are slightly different. The kind of claims of the players and the relationship between the given claims and the given resources available, on one hand, and the specific way of evaluating the value of each possible coalition, on the other hand, determine the particular type of game which has to be solved in each of the three ancient problems mentioned.

In order to apply the Shapley value of the players, the generalized characteristic function of each game has to be defined. The essential point is that the value of this generalized characteristic function assigned to a player has to reflect the maximum claim this player can make, subject to the restrictions induced by the relationship between all the claims and the given estate. Each player with a claim smaller than the estate may ask for that claim but, as discussed in Sect. 2.2, if no claim is smaller than the current portion of the estate still available, the respective players have to share equally this surplus. As a consequence, when the generalized characteristic function of the corresponding game is defined, we have to make a distinction between the players whose claims are smaller than the estate and those whose claims are not smaller than the estate.

The value of each proper potential coalition, however, is not uniquely determined by the given claims and estate. It may be calculated either by summing up the claims of the members of the respective coalition or by taking the maximum of these claims, to the extent to which they do not exceed the estate. The decision based on the first kind of computation induces a cumulative game. The decision based on the second kind of computation induces a maximal game. In a cumulative game the players do not cooperate and are not willing to share or reach a compromise when they join a coalition. In a maximal game the players are supposed to be willing to compromise, and share parts of their claims when they join a coalition. The ancient division of the estate shown in Tables 1-3 may be obtained by using the Shapley value, as the unique mathematical tool, if the game corresponding for the three wives problem is cumulative and the game corresponding to the rights arbitration problem is maximal. Finally, the contested garment problem is very simple and contains no proper coalitions; it may be solved, as a simple two-person game, in one straightforward step. 
Section 2 contains the general, one-step solutions of the three ancient problems, viewed as $n$-person games. Section 3 contains the iterative, step-by-step solution of the bankruptcy problem and the right arbitration problem. It is shown that the Shapley value method implies the well-known Aumann-Maschler iterative solution of the bankruptcy problem.

Examining today the old solutions given to the three ancient problems, the numbers from Table 1 look like they were obtained by Rabbi Nathan using the Shapley value, knowing that the three wives hated each other, whereas the numbers from Table 3 look like they were obtained by Abraham Ibn Ezra using the same Shapley value but assuming that the four brothers were willing to compromise and share parts of their claims!

Acknowledgements The author is indebted to Dr. Wiebe van der Hoek, the Editor-in-Chief, for his constructive comments, and to the two referees for their criticism and very valuable suggestions that have essentially contributed to make the final version of the paper better. The author thanks Dr. Eli Brettler, who gave him the seminal paper Aumann and Maschler (1985).

\section{References}

Alcade, J., Marco, M. del C., \& Silva, J. A. (2005). Bankruptcy games and the Ezra's proposal. Economic Theory, 26, 103-114.

Aumann, R. J., \& Maschler, M. (1985). Game-theoretic analysis of a bankruptcy problem from the Talmud. Journal of Economic Theory, 36, 195-213.

De Mesnard, L. (2008). On the Talmud division: Equity and robustness. Congrès AFSE (Social Science Research Network SSRN), 1-27.

Gura, E.-Y. (2009). Insights into the game theory: An alternative mathematical experience. In Quaderni di Ricerca Didattica. G.R.I.M. (Department of Mathematics, University of Palermo, Italy), n. 19, pp. $172-183$.

Moulin, H. J. (1992). An application of the Shapley value to fair division with money. Econometrics, 60, 1331-1349.

Moulin, H. J. (2003). Fair division and collective welfare. Cambridge, MA: MIT Press.

O'Neill, B. (1982). A problem of rights arbitration from the Talmud. Mathematical Social Sciences, 2, 345-371.

Shapley, L. S. (1953). A value for $n$-person games. Annals of Mathematical Studies, 28, 307-317.

Thomson, W. (2003). Axiomatic and game-theoretic analysis of bankruptcy and taxation problems: A survey. Mathematical Social Sciences, 45, 249-297.

Winston, W. L. (1994). Operations research. Applications and algorithms (3rd ed.). Belmont CA: Duxbury Press, Wadsworth Publishing Co. 